\title{
An Interval NLPV Parity Equations Approach for Fault Detection and Isolation of a Wind Farm
}

\author{
Joaquim Blesa, Pedro Jiménez, Damiano Rotondo, Fatiha Nejjari, and Vicenç Puig
}

\begin{abstract}
In this paper, the problem of fault diagnosis of a wind farm is addressed using interval nonlinear parameter varying (NLPV) parity equations. Fault detection is based on the use of parity equations assuming unknown but bounded description of the noise and modeling errors. The fault detection test is based on checking the consistency between the measurements and the model by finding if the formers are inside the interval prediction bounds. The fault isolation algorithm is based on analyzing the observed fault signatures on-line, and matching them with the theoretical ones obtained using structural analysis. Finally, the proposed approach is tested using the wind farm benchmark proposed in the context of the wind farm FDI/FTC competition.
\end{abstract}

Index Terms-Fault diagnosis, Wind farm, Interval NLPV parity equations.

\section{INTRODUCTION}

Wind energy has gained lot of interest in the last years as a promising and abundant source for green and sustainable energy. As a result of wind turbine technology development, wind farms of increasing size are being established for largescale harvesting of this abundant renewable energy source [1]-[3]. However, wind farms are characterized by high manufacturing and maintenance costs and challenging reliability and lifetime issues [4]-[7]. Introducing fault diagnosis and fault tolerant control (FTC) techniques allows to improve the reliability and reduce the maintenance cost of wind farms [8].

In recent years, the problem of fault diagnosis and FTC of wind turbines has become an important topic of research. For example, [9] has discussed the design, implementation, experimental validation and performances of an FPGA-based real-time power converter failure diagnosis for three-leg fault tolerant converter topologies used in wind energy conversion systems. [10] has described a wind turbine condition monitoring technique that used the generator output power and rotational speed to derive a fault detection signal. Both mechanical and electrical fault-like perturbations were successfully detected using an adaptive filter. [11] has proposed a method

Manuscript received March 31, 2014; revised June 28, 2014, August 22, 2014, October 8, 2014 and November 15, 2014; accepted December 2, 2014.

This work has been funded by the Spanish MINECO through the project CYCYT SHERECS (ref. DPI2011-26243), by the European Commission through contract i-Sense (ref. FP7-ICT-2009-6-270428), by AGAUR through the contracts FI-DGR 2013 (ref. 2013FIB00218) and FI-DGR 2014 (ref. 2014FI_B1 00172) and by the DGR of Generalitat de Catalunya (SAC group Ref. 2014/SGR/374).

The authors are with the Research Center for Supervision, Safety and Automatic Control (CS2AC), Universitat Politècnica de Catalunya (UPC), Edifici TR11, Rambla Sant Nebridi 10, 08222 Terrassa, Spain (e-mails: name.surname@upc.edu). Joaquim Blesa and Vicenç Puig are also with the Institut de Robòtica i Informàtica Industrial, CSIC-UPC, Carrer de Llorens i Artigas, 4-6, 08028 Barcelona, Spain. consisting of appropriate current frequency and amplitude demodulation algorithms for bearing fault diagnosis using only stator current measurements. [12] has addressed open-circuit fault diagnosis in the power converters of a permanent magnet synchronous generator drive for wind turbine applications. The diagnosis method allowed real-time detection and localization of multiple open-circuit faults.

In [13], a benchmark model for fault detection and isolation (FDI) and FTC of wind turbines, describing a realistic three blade horizontal variable speed wind turbine with a full scale converter coupling and a rated power of $4.8 \mathrm{MW}$, has been presented. Some solutions have been proposed recently as a part of an international competition, covering different types of FDI methods, and showing interesting potential for usage in the wind turbine application (see [13] for a summary of the main results). In [14], a combination of a diagnosis observer and a Kalman filter for the generation of the residuals, that are later evaluated using their statistical properties, has been proposed. A bank of residual generators based on dual sensor redundancy has also been designed for fault isolation purpose. [15] has presented a diagnosis strategy based on fuzzy models in order to detect and isolate actuator and sensor faults in the converter. An FDI system has been designed by applying a generic automated method that did not require a specific adaptation to the benchmark in [16]. The method consisted of three steps: generation of candidate residual generators, residual generator selection, and diagnosis test construction. In [17], set-theoretic methods describing invariant sets under both healthy and faulty conditions have been implemented. By analysing the relative information with respect to these sets, fault diagnosis has been performed. In [18], fault detection was based on the use of interval observers, and fault isolation was performed by matching the observed fault signatures with the theoretical ones, obtained using structural analysis and a row-reasoning scheme.

In some of these works, the fault detection problem has been addressed using model-based approaches that are based on analytical redundancy and checking the consistency of the observed behavior with respect to the modeled one. This consistency checking is based on computing the difference, called residual, between the value predicted from the model and the real value measured by the sensors. In case a discrepancy is detected, a fault in the system is indicated. Otherwise, it is considered that the system is working properly.

In the case of linear time invariant (LTI) systems, modelbased fault detection theory is well developed [19]-[22]. However, since these methods are based on LTI lumped parameter models, they are only valid around a given op- 
erating point. In order to use these simplified models in large operating conditions, the influence of the operating point in the parameters of the LTI model should be taken into account. In this paper, the use of nonlinear parameter varying (NLPV) models is proposed in order to consider the variation of the parameters with the operating point. This type of models adds a nonlinear dynamic map to the classical linear parameter varying (LPV) model, which takes into account the scheduling variables available for measurement, resulting in a hybrid linear/nonlinear model. In this way, the advantages of a parametrically varying structure and the generality of the NARMAX (nonlinear autoregressive exogenous moving average) class [23] are combined. It should be stated that the Takagi-Sugeno (TS) paradigm [24], [25] could be considered instead of the LPV one. According to [26]-[28], LPV models and TS models are very similar. Thus, the results obtained by adding a nonlinear dynamic map to a TS model would be very close to the ones obtained in this work.

Fault detection methods based on mathematical models are always affected by modeling errors. A fault detection algorithm able to handle uncertainty is called robust, and its robustness represents the degree of sensitivity to faults compared to the degree of sensitivity to uncertainty [20]. One of the most developed families of approaches to deal with model uncertainty, called active, is based on generating residuals, which are insensitive to uncertainty (modeling errors and disturbances), while at the same time sensitive to faults using some decoupling method [20]. On the other hand, there is a second family of approaches, called passive, which enhances the robustness of the fault detection system at the decision-making stage using an adaptive threshold [29]-[32].

In this paper, the uncertainty will be considered in the parameters and in the delay of the NLPV model, bounding their values by intervals. The robustness in fault detection is achieved by means of the passive approach based on an adaptive threshold generated by considering the set of model responses obtained by varying the uncertain parameters within their intervals and the bounded description of the noise. Using adaptive thresholds has many advantages in comparison to the fixed ones. In case of using fixed thresholds, those should be fixed considering the worst-case uncertainty scenario in time. On the other hand, adaptive thresholds allow considering at each time instant a different threshold according to the input.

The aim of this work is to address the problem of fault diagnosis of a wind farm, which is a very recent field of research [33]. This problem has been addressed only in few works as in [8], where an evolving classification method has been proposed. However, among the three types of fault proposed in the benchmark [33], i.e. (1) debris build-up on the wind turbine blades, (2) misalignment of one or more blades originated at the time of installation of the wind turbine, and (3) change in the drive train damping due to wear and tear, [8] has considered only the first type of fault. On the contrary, in this work, all the three types of faults proposed by [33] are considered.

In this work, the fault diagnosis problem is tackled using interval NLPV parity equations [34]. Fault detection is based on the use of parity equations, assuming an unknown but bounded description of the noise and modeling errors. The fault detection test is based on checking the consistency between the measurements and the model, finding out if the formers are inside the interval prediction bounds. The fault isolation algorithm is based on analyzing the observed fault signatures on-line, and matching them with the theoretical ones obtained using structural analysis. The approach is tested using the wind farm benchmark proposed in the context of the wind farm FDI/FTC competition [33].

The paper is organized as follows. Section II presents a method for using interval NLPV parity equations in fault detection, as well as the estimation of the parametric uncertainty intervals. In Section III, the wind farm benchmark is presented and the fault diagnosis approach based on interval NLPV parity equations is applied. The results obtained applying the proposed method to the wind farm benchmark are shown in Section IV. Finally, in Section V, the main conclusions are given.

\section{FAULT DETECTION USING INTERVAL NLPV PARITY EQUATIONS}

\section{A. Interval NLPV model}

Let us assume that the system can be expressed by means of the following NLPV model in discrete time:

$$
y(k)=F\left(k, \theta\left(p_{k}\right)\right)+e(k)=\hat{y}(k)+e(k)
$$

where:

- $F\left(k, \theta\left(p_{k}\right)\right)$ is the regressor NARMAX function used to compute the estimation $\hat{y}(k)$ which, in general, is assumed to be nonlinear in the parameters $\theta\left(p_{k}\right)$ and can contain any function of inputs $u(k)$ and estimated outputs $\hat{y}(k)$;

- $p_{k} \triangleq p(k)$ is a vector of measurable process variables that defines the system operating point;

- $\theta\left(p_{k}\right) \in \Theta_{k}$ is the parameter vector of dimension $n_{\theta} \times 1$, whose values can vary according to the operating point;

- $\Theta_{k}$ is the set that bounds the uncertainty in the parameter varying values, that also varies according to the operating point. In particular, in this paper, the set of uncertain parameters is bounded by an interval box centered in the nominal parameter values:

$$
\Theta_{k} \triangleq\left[\underline{\theta}_{1}\left(p_{k}\right), \bar{\theta}_{1}\left(p_{k}\right)\right] \times \ldots \times\left[\underline{\theta}_{n_{\theta}}\left(p_{k}\right), \bar{\theta}_{n_{\theta}}\left(p_{k}\right)\right]
$$

where:

$$
\begin{aligned}
& \underline{\theta}_{i}\left(p_{k}\right) \triangleq \theta_{i}^{0}\left(p_{k}\right)-\lambda_{i}(k) \quad i=1, \ldots, n_{\theta} \\
& \bar{\theta}_{i}\left(p_{k}\right) \triangleq \theta_{i}^{0}\left(p_{k}\right)+\lambda_{i}(k)
\end{aligned}
$$

being $\theta_{i}^{0}\left(p_{k}\right)$ the nominal parameter values that follow some known function $f\left(p_{k}\right)$, and $\lambda_{i}(k) \geq 0$ the bounds on the parameter uncertainties;

- $e(k)$ is an additive error that includes noise and discretization errors, which is unknown but assumed to be bounded by a constant $|e(k)| \leq \sigma$ 


\section{B. Interval fault detection using NLPV parity equations}

The principle of model-based fault detection using parity equations is to test whether or not the measured inputs and outputs of the system are consistent with the behavior described by a model of the faultless system. If an inconsistency is detected, a fault is indicated. The residual usually describes the consistency check between the real behavior $y(k)$ and the predicted one $\hat{y}(k)$, as follows:

$$
r(k)=y(k)-\hat{y}(k)
$$

where $\hat{y}(k)=F\left(k, \theta\left(p_{k}\right)\right)$, considering the system described by (1).

Ideally, residuals should be affected only by the faults. However, the presence of disturbances, noise and modeling errors causes the residuals to become non-zero, interfering with the fault detection. Therefore, the fault detection procedure must be robust against these undesired effects [20]. In this work, only noise and modeling errors will be considered in the robust fault detection method, following the passive approach recalled in the introduction.

In the case of modeling a dynamical system using the interval NLPV model (1), the predicted output behavior can be bounded at each iteration by an interval $[\hat{y}(k), \overline{\hat{y}}(k)]$, computed by solving the two following optimization problems ${ }^{1}$ :

$$
\begin{aligned}
& \overline{\hat{y}}(k)=\max \left(F\left(k, \theta\left(p_{k}\right)\right)\right) \text { s.t. } \theta\left(p_{k}\right) \in \Theta_{k} \\
& \underline{\hat{y}}(k)=\min \left(F\left(k, \theta\left(p_{k}\right)\right)\right) \text { s.t. } \theta\left(p_{k}\right) \in \Theta_{k}
\end{aligned}
$$

Then, the fault detection test is based on propagating the parameter uncertainty and the additive error bounds, including noise and discretization errors, to the estimation and checking if:

$$
y(k) \in[\underline{\hat{y}}(k)-\sigma, \overline{\hat{y}}(k)+\sigma]
$$

holds or not. In case it does not hold, a fault can be indicated.

Test (5) can be formulated alternatively as:

$$
r^{0}(k) \in[\underline{r}(k), \bar{r}(k)]=\left[\underline{\hat{y}}(k)-\hat{y}^{0}(k)-\sigma, \overline{\hat{y}}(k)-\hat{y}^{0}(k)+\sigma\right]
$$

with $r^{0}(k)=y(k)-\hat{y}^{0}(k)$, where $\hat{y}^{0}(k)$ is the nominal prediction given by $F\left(k, \theta^{0}\left(p_{k}\right)\right)$.

\section{Fault detection for uncertain varying time delay systems}

In case that (1) is used to represent a system with transport delay, this delay can also vary with the operating point and can be characterized by some measured variables $p_{k}$. Considering such varying transport delay $\tau\left(p_{k}\right)$ in the model (1), the output prediction can be expressed as:

$$
\hat{y}(k)=F\left(k, \theta\left(p_{k}\right), d\left(p_{k}\right)\right)
$$

with $d\left(p_{k}\right)=\left\lceil\frac{\tau\left(p_{k}\right)}{T_{s}}\right\rceil$, where \lceil\rceil denotes the nearest integer and $T_{s}$ is the sampling time. Some uncertainty $\lambda_{\tau}$ in the delay is also considered such that:

$$
\tau\left(p_{k}\right) \in\left[\tau_{0}\left(p_{k}\right)-\lambda_{\tau}, \tau_{0}\left(p_{k}\right)+\lambda_{\tau}\right]
$$

\footnotetext{
${ }^{1}$ These optimization problems are solved by means of interval arithmetic evaluation [35].
}

where the nominal delay satisfies: $\tau_{0}\left(p_{k}\right)>\lambda_{\tau}$ and $\tau_{0}\left(p_{k}\right), \lambda_{\tau} \in \mathbb{R}^{+}$.

Then, as suggested in [36], the interval for the predicted output given by (7) can be expressed as follows:

$$
\begin{aligned}
& \overline{\hat{y}}(k)=\max _{\theta\left(p_{k}\right) \in \Theta_{k}, d\left(p_{k}\right) \in\left\{d\left(p_{k}\right), \ldots, \overline{d\left(p_{k}\right)}\right\}} F\left(k, \theta\left(p_{k}\right), d\left(p_{k}\right)\right) \\
& \underline{\hat{y}}(k)=\min _{\theta\left(p_{k}\right) \in \Theta_{k}, d\left(p_{k}\right) \in\left\{\underline{\left.d\left(p_{k}\right), \ldots, \overline{d\left(p_{k}\right)}\right\}}\right.} F\left(k, \theta\left(p_{k}\right), d\left(p_{k}\right)\right)
\end{aligned}
$$

with $\underline{d\left(p_{k}\right)}=\left\lceil\frac{\tau_{0}\left(p_{k}\right)-\lambda_{\tau}}{T_{s}}\right\rceil$ and $\overline{d\left(p_{k}\right)}=\left\lceil\frac{\tau_{0}\left(p_{k}\right)+\lambda_{\tau}}{T_{s}}\right\rceil$.

\section{Model Calibration using Parity Equations}

One of the key points in model based fault detection is how models are built and their uncertainty is estimated. In this paper, it is assumed that the regressor function $F$ in (1), the nominal parameter $\theta^{0}\left(p_{k}\right)$ and the nominal delay $\tau_{0}\left(p_{k}\right)$ are given by the physical modeling of the system. Hence, the parameters to be calibrated are the parameter uncertainty sets $\Theta_{k}$ given by $\lambda_{i}(k)$ for $i=1, \ldots, n_{\theta}$ and the delay uncertainty $\lambda_{\tau}$. The delay uncertainty $\lambda_{\tau}$ can be determined considering the input process signal to be white noise and carrying out the study of the independence between the input and output process signals using confidence intervals (usually, $95 \%$ or 99\%) as in [36].

On the other hand, given $N$ measurements of outputs and inputs from a non-faulty scenario rich enough from the identifiability point of view, the uncertain parameter estimation algorithm proceeds through the following optimization problem that can be solved off-line by bi-level optimization [37]:

$\min \alpha$

subject to :

$$
\begin{aligned}
& y(k) \in[\underline{\hat{y}}(k)-\sigma, \overline{\hat{y}}(k)+\sigma] \quad k=1, \ldots, N \\
& \underline{\hat{y}}(k)=\max _{\theta\left(p_{k}\right) \in \Theta_{k}, d\left(p_{k}\right) \in\left\{d\left(p_{k}\right), \ldots, \overline{d\left(p_{k}\right)}\right\}} F\left(k, \theta\left(p_{k}\right), d\left(p_{k}\right)\right) \\
& \hat{y}(k)=\min _{\theta\left(p_{k}\right) \in \Theta_{k}, d\left(p_{k}\right) \in\left\{d\left(p_{k}\right), \ldots, \overline{d\left(p_{k}\right)}\right\}} F\left(k, \theta\left(p_{k}\right), d\left(p_{k}\right)\right) \\
& \Theta_{k} \triangleq\left[\underline{\theta}_{1}\left(p_{k}\right), \bar{\theta}_{1}\left(p_{k}\right)\right] \times \ldots \times\left[\underline{\theta}_{n_{\theta}}\left(p_{k}\right), \bar{\theta}_{n_{\theta}}\left(p_{k}\right)\right] \\
& \underline{\theta}_{j}\left(p_{k}\right)=\theta_{j}^{0}\left(p_{k}\right)-\lambda_{j}(k) \\
& \bar{\theta}_{j}\left(p_{k}\right)=\theta_{j}^{0}\left(p_{k}\right)+\lambda_{j}(k) \\
& \lambda_{j}(k)=\alpha \theta_{j}^{0}\left(p_{k}\right), j=1, \ldots, n_{\theta}
\end{aligned}
$$

\section{WIND FARM BENCHMARK}

The wind farm benchmark, described in detail in [33], is made up by 9 wind turbines in a square grid layout (see Fig. 1). The distance between the wind turbines in both directions is $7 L$, where $L$ is the rotor diameter. Two measuring masts are located in front of the wind farm, one for each possible wind direction considered in the benchmark. The distance between the measuring mast and the wind farm is $10 L$. Each turbine is a generic 4.8 MW wind turbine, as the one described in [13], and is numbered using the corresponding row and column in the wind farm ${ }^{2}$. Each wind turbine is driven by the wind farm

${ }^{2}$ Along the paper subscript indexes $i$ and $j$, or particular numbers $1,2,3$, separated by comma will be added to variables to denote the particular wind turbine they refer to, following the nomenclature introduced in Fig. 1, i.e $i$ for row and $j$ for column. 
controller that provides a power reference.

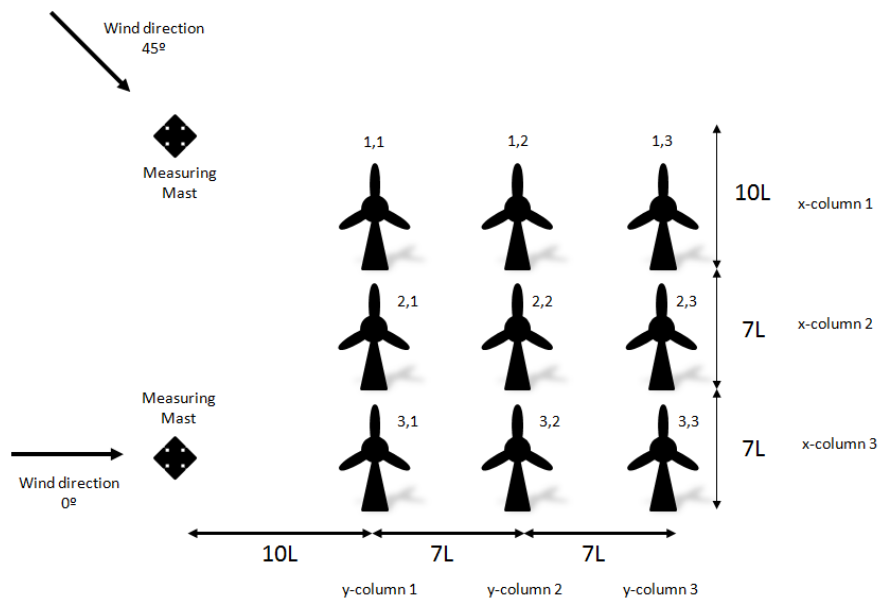

Fig. 1. Illustration of the layout of the benchmark wind farm.

\section{A. Wind Farm Model}

The benchmark of the wind farm model consists of three parts:

- the wind and wake model: the wind sequence at the measuring mast is the same in both the scenarios considered in the benchmark, but the wind direction can be either $0^{\circ}$ or $45^{\circ}$. The wind sequence is $4400 \mathrm{~s}$ long and its mean value increases from $5 \mathrm{~m} / \mathrm{s}$ to $15 \mathrm{~m} / \mathrm{s}$, with a peak around $23 \mathrm{~m} / \mathrm{s}$, so that it contains the full operating range of the wind turbines. The wind sequence is delayed by the distance between the different points in the wind farm and the mean wind speed. The wake is modeled by a wind deficit among the wind turbines by a factor of 0.9 .

- the wind turbine model: each turbine is a three bladed horizontal axis pitch controlled variable speed wind turbine. Its model provides three outputs (generated electrical power $P$, collective pitch angle $\beta$ and generator speed $\omega_{g}$ ) from two inputs (wind speed $v_{w}$ and power reference $P_{r}$ ). Since the wind turbine controller regulates the pitch angles to be the same, only one measured pitch angle is provided. The model is based on the wind turbine model included in the wind farm developed in [38], and describes the closed-loop turbine behavior comprising a power model, a pitch model and a generator speed model. All the models rely on approximating static functions and relations in frequency domain. The non-linearity of the models is due to the aerodynamic part of the wind-turbines as well as some changes of the behavior according to the wind turbine region of operation as described in [33].

- the wind farm controller: it is implemented in discrete time with a sample time of $10 s$ and gives the power reference to each individual wind turbine based on the power requested by the operator, that can take any value between 0 and $43.6 \mathrm{MW}$, the latter value corresponding to the power given by the nine wind turbines when they are working at their nominal power 4.8 MW.

\section{B. Fault Scenarios}

Three different faults are considered in the benchmark, affecting the three measured variables, that are the power $P$, the pitch angle $\beta$ and the generator angular velocity $\omega_{g}$. The faults are hard to detect at a wind turbine level, but can be detected at the wind farm level comparing the performances of the other wind turbines.

Fault 1: debris build-up on the wind turbine blades, changing the aerodynamics of the wind turbine, lowering the maximum obtained power [39].

Fault 2: misalignment of one or more blades originated at the time of installation of the wind turbine. This fault leads to an offset between the measured and the actual pitch angle for one or more blades. Since the controller will compensate the offset by changing the pitch angles in the faulty turbine, a difference with respect to the angle of other wind turbines operating with the same wind speed will appear.

Fault 3: change in the drive train damping due to wear and tear. At wind turbine level, this fault could be detected monitoring changes in the frequency spectra of the vibration measurements. However, the solutions provided in the Wind Turbine FDI competition suggest that it is hard to detect changes in the drive train damping only at the wind turbine level [40]. Hence, it is interesting to investigate if this fault could be detected at the wind farm level.

\section{Wind Estimation}

The wind sequence in each wind turbine is estimated by considering the distance between the measuring mast and the wind turbine itself. The delay in the wind sequence is the distance divided by the mean wind velocity, while the wake is modeled by a wind deficit between the wind turbines by a factor of 0.9 .

\section{Models for fault detection}

The models considered for FDI in the wind farm take into account the temporal and spatial redundancy existing in the wind farm.

The temporal redundancy in each wind turbine can be taken into account by considering the estimated wind received by each wind turbine, the power reference supplied by the wind farm controller and the approximated model that relates the measured variables to estimate the power $P$, pitch angle $\beta$ and angular velocity $\omega_{g}$.

The wind speed at the turbine $i, j v_{w_{i, j}}(t)$ can be estimated from the wind speed measured in a wind mast $v_{w}(t)$ with:

$$
v_{w_{i, j}}(t)=f_{v_{w_{i, j}}}\left(v_{w}\left(t-\tau_{i, j}(t)\right)\right)+e_{w_{i, j}}(t)=\hat{v}_{w_{i, j}}+e_{w_{i, j}}(t)
$$

where $f_{v_{w_{i, j}}}$ is a function that computes the wind deficit between the wind turbines, $e_{w_{i, j}}(t)$ is the wind estimation error in the turbine $i, j$, considered unknown but bounded $\left|e_{w_{i, j}}(t)\right| \leq \sigma_{w_{i, j}}$, and $\tau_{i, j}$ is the wind transport delay from the wind mast to the turbine $i, j$, that can be calculated as:

$$
\tau_{i, j}(t)=f_{D_{i, j}}\left(v_{w}^{0}(t)\right)+e_{\tau_{i, j}}(t), \quad\left|e_{\tau_{i, j}}(t)\right| \leq \lambda_{\tau_{i, j}}
$$

where $v_{w}^{0}(t)$ is the mean wind speed at the wind mast point (see Fig. 1), obtained by filtering the wind measurement $v_{w}(t)$. 
The bounded additive error $e_{\tau_{i, j}}(t)$ is the error in the delay estimation. In the wind and wake models used in this work, for the wind scenario 1 ( 0 deg wind direction), the transport delays $\tau_{i, j}(t)$ can be estimated as follows:

$$
\begin{aligned}
& f_{D_{i, 1}}\left(v_{w}^{0}(t)\right)=\frac{10 L}{v_{w}^{0}(t)}, \quad \forall i=1,2,3 \\
& f_{D_{i, 2}}\left(v_{w}^{0}(t)\right)=\frac{10 L}{v_{w}^{0}(t)}+\frac{7 L}{0.9 v_{w}^{0}(t)}, \quad \forall i=1,2,3 \\
& f_{D_{i, 3}}\left(v_{w}^{0}(t)\right)=\frac{10 L}{v_{w}^{0}(t)}+\frac{7 L}{0.9 v_{w}^{0}(t)}+\frac{7 L}{0.81 v_{w}^{0}(t)}, \quad \forall i=1,2,3
\end{aligned}
$$

The estimations of the power, pitch angle and angular velocity variables can be computed as:

$$
\begin{gathered}
\hat{P}_{i, j}(k)=F_{P_{i, j}}\left(k, d_{i, j}\left(p_{k}\right), \theta_{P_{i, j}}\left(p_{k}\right), P_{r_{i, j}}(k)\right) \\
\hat{\beta}_{i, j}(k)=F_{\beta_{i, j}}\left(k, d_{i, j}\left(p_{k}\right), \theta_{\beta_{i, j}}\left(p_{k}\right), P_{r_{i, j}}(k), \hat{\beta}_{i, j}(k-1)\right) \\
\hat{\omega}_{g i, j}(k)=F_{\omega_{g i, j}}\left(k, d_{i, j}\left(p_{k}\right), \theta_{\omega_{g i, j}}\left(p_{k}\right), P_{r_{i, j}}(k)\right)
\end{gathered}
$$

where $F_{P_{i, j}}, F_{\beta_{i, j}}$ and $F_{\omega_{g i, j}}$ are nonlinear functions that can be derived from the simplified model proposed in [33] after time discretisation. These functions have the same input: the power reference $P_{r_{i, j}(k)}$ supplied by the controller and filtered to avoid abrupt changes. The LPV parameters are: $\theta_{P_{i, j}}\left(p_{k}\right)=$ $\theta_{\omega_{g i, j}}\left(p_{k}\right)=\hat{P}_{w_{i, j}}(k)$ and $\theta_{\beta_{i, j}}\left(p_{k}\right)=\left(P_{a_{i, j}}(k), h_{i, j}(k)\right)^{T}$ that correspond to the dynamically available power $\hat{P}_{w_{i, j}}(k)$, the theoretical available power $P_{a_{i, j}}(k)$ and the nonlinear pitch parameter $h_{i, j}(k)$. All of these parameters can be computed with the wind measurement $v_{w}(k)$ as described in [33], considering the wind deficit defined by $f_{v_{w i, j}}$ and the discrete time delay $d_{i, j}\left(p_{k}\right)$ that can be computed as:

$$
d_{i, j}\left(p_{k}\right)=\left\lceil\frac{f\left(D_{i, j}, v_{w}^{0}(k)\right)}{T_{S}}\right\rceil
$$

Then, the scheduling variables are:

$$
p_{k}=\left(v_{w}^{0}(k), v_{w}(k)\right)^{T}
$$

$\hat{P}_{w_{i, j}}(k)$ can be computed using a nonlinear function and a first order low pass filter defined in (6) and (7) of [33]. $P_{a_{i, j}}(k)$ is obtained from the nonlinear function (12) of [33]. $h_{i, j}(k)$ is a nonlinear function defined in equation (13) of [33]. Finally, the nonlinear function $F_{P_{i, j}}$ is obtained including $\hat{P}_{w_{i, j}}(k)$ in the nonlinear equation (9) and combined with equation (10) of [33]. The nonlinear function $F_{\beta_{i, j}}$ is obtained computing the first order transfer function (11) of [33], where the input is the pitch reference obtained with the ratio between the available power $P_{a_{i, j}}(k)$ and the reference power $P_{r_{i, j}}(k)$. If the available power is lower than the reference power, $h_{i, j}(k)$ is used in (13) to compute the pitch reference. The nonlinear function $F_{\omega_{g i, j}}$ is obtained including the parameters $\hat{P}_{w_{i, j}}(k)$ in the nonlinear equation (9) combined with equation (14) of [33].

\section{E. Set of considered residuals}

Subtracting estimations (13)-(15) from real data measurements, three residuals are obtained for every wind turbine, resulting in 27 residuals, e.g. the temporal residual of the generated power in turbine 1,1 would be $\mathrm{b}^{3}$ :

$$
r_{T} P_{1,1}(k)=P_{1,1}(k)-\hat{P}_{1,1}(k)
$$

The main drawback of these 27 temporal parity equations is that they depend directly on the scheduling variable which, in this case, is the wind, that is measured with a high noise level, leading to a big wind speed estimation error bound $\sigma_{w_{i, j}}$ in (10). On the other hand, the spatial redundancy is based on comparing those wind turbines that are in the same wind column, i.e. that receive approximately the same wind. This allows generating another set of analytical redundancy relations that are less dependent on the wind measurements. For example, in wind scenario 1 (wind direction $0 \mathrm{deg}$ ), the wind for all the wind turbines that are in the same $y$-column (see Fig. 1) is expected to be approximately the same at every instant $k$, that implies:

$$
v_{w_{j}}(k) \approx v_{w_{1, j}}(k) \approx v_{w_{2, j}}(k) \approx v_{w_{3, j}}(k) \quad j=1,2,3 \quad \forall k
$$

Then, in this wind scenario, there are three wind columns that match with the y-columns of the wind farm. On the other hand, if the reference power is the same for all the generators in the wind farm, as it usually is the case, the following spatial relations could be considered among wind turbines:

$$
\begin{array}{rrr}
P_{1, j}(k) \approx P_{2, j}(k) \approx P_{3, j}(k) & j=1,2,3 & \forall k \\
\beta_{1, j}(k) \approx \beta_{2, j}(k) \approx \beta_{3, j}(k) & j=1,2,3 & \forall k \\
\omega_{g 1, j}(k) \approx \omega_{g 2, j}(k) \approx \omega_{g 3, j}(k) & j=1,2,3 & \forall k
\end{array}
$$

In practice, the fact that the wind magnitude will not be exactly the same for all the wind turbines in the same wind column can be taken into account by including some bounded additive error ${ }^{4}$, as follows:

$$
\begin{aligned}
& v_{w_{1, j}}(k)=v_{w_{j}}(k)+e_{w_{1, j}}(k) \quad\left|e_{w_{1, j}}(k)\right| \leq \sigma_{w_{j}} \\
& v_{w_{2, j}}(k)=v_{w_{j}}(k)+e_{w_{2, j}}(k) \quad e_{w_{2, j}}(k) \leq \sigma_{w_{j}} \\
& v_{w_{3, j}}(k)=v_{w_{j}}(k)+e_{w_{3, j}}(k) \quad\left|e_{w_{3, j}}(k)\right| \leq \sigma_{w_{j}}
\end{aligned}
$$

and (18) would lead to the following 9 spatial residuals ${ }^{5}$ :

$$
\begin{array}{ll}
r_{S} P_{1-2, j}(k)=P_{1, j}(k)-P_{2, j}(k) & j=1,2,3 \\
r_{S} P_{1-3, j}(k)=P_{1, j}(k)-P_{3, j}(k) & j=1,2,3 \\
r_{S} P_{2-3, j}(k)=P_{2, j}(k)-P_{3, j}(k) & j=1,2,3
\end{array}
$$

${ }^{3}$ Temporal residuals will be denoted as $r_{T} M_{i, j}$, where $r_{T}$ denotes temporal residual, $M$ the considered variable $\left(P, \beta\right.$ or $\left.\omega_{g}\right)$ and indexes $i, j$ refer to the particular turbine.

${ }^{4}$ The assumption that the wind is approximately the same is reasonable in many situations, in particular at a sufficient height above the earth surface, far from complex terrains, such that the interaction of other elements, e.g. sea waves, can be neglected. In particular, [41] has shown that there is some spatial variation in the average inflow wind speed between the average wind speed at the meteo mast and the individual turbines, causing differences of the order of $1 \mathrm{~m} / \mathrm{s}$ for their case study.

${ }^{5}$ Spatial residuals will be denoted as $r_{S} M_{l-h, j}$ where $r_{S}$ denotes spatial residual, $M$ the considered variable $\left(P, \beta\right.$ or $\left.\omega_{g}\right)$, indexes $l$ and $h$ indicate the elements of the wind column that are subtracted and index $j$ denotes the wind column. 
The same relations are obtained for the pitch and the angular velocity. This means that 27 additional parity equations are added to the 27 temporal parity equations. On the other hand, in wind scenario 2 (45 deg wind direction), there are 5 different wind columns. The first and the last wind columns contain one single element, i.e. wind turbine 1,1 and wind turbine 3,3 , respectively. Hence, it is not possible to obtain spatial relations for these turbines in this wind scenario. Regarding the other wind columns, the following speed relations can be deduced:

$$
\begin{aligned}
& \text { wind column } 2\left(45^{\circ}\right): v_{w_{2,1}}(k) \approx v_{w_{1,2}}(k) \\
& \text { wind column } 3\left(45^{\circ}\right): v_{w_{3,1}}(k) \approx v_{w_{2,2}}(k) \approx v_{w_{1,3}}(k) \\
& \text { wind column } 4\left(45^{\circ}\right): v_{w_{3,2}}(k) \approx v_{w_{2,3}}(k) \quad \forall k
\end{aligned}
$$

Hence, 15 additional spatial parity equations can be obtained in wind scenario 2.

The main advantage of the spatial relations versus the temporal ones is that the formers are only affected by the maximum difference of the wind speed in the same wind column $\sigma_{w_{j}}$, that is supposed to be smaller than the wind speed estimation error bound in the wind turbines $\sigma_{w_{i, j}}$. This is due to the fact that the bound $\sigma_{w_{i, j}}$ is affected by the uncertainty in transport delay $\lambda_{\tau_{i j}}$ introduced in (11).

Remark: Since the spatial relations depend on the wind direction, they have to be reconfigured according to the wind direction, obtained by means of a sensor or estimated with the available measurements. In this work, only $0 \mathrm{deg}$ and 45 deg wind directions have been considered, as proposed in the wind farm benchmark [33]. Then, when the system will detect a wind direction close to $0 \mathrm{deg}$ or $45 \mathrm{deg}$, it will benefit from spatial relations. This is specially useful in wind case 1 ( $0 \mathrm{deg})$, since wind farms are usually installed in the predominant wind directions. The generation of spatial residuals for a generic wind direction will be considered in a future work.

\section{F. Fault diagnosis}

Fault 1 influences the power residuals, Fault 2 influences the pitch angle residuals and, finally, Fault 3 influences the power and the generator speed residuals.

According to the wind farm benchmark, the effect of Fault 3 is the increase of the amplitude of the drive train oscillation that has a frequency of $10 \mathrm{~Hz}$, as described in [33], while Faults 1 and 2 are low frequency faults. This fact, and the need of filtering the residuals in order to decrease the effect of measurement noises, lead to filter the power residuals with low pass (LP) and band pass (BP) filters so as to better detect Fault 1 and Fault 3, respectively. On the other hand, low pass and band pass filters have been used in pitch angle and speed residuals, in order to enhance the detectability of Faults 2 and 3 , respectively.

Table I shows the part of the theoretical signature matrix that can be obtained from the temporal residuals of a particular wind turbine $i, j$. The subscripts $L P$ and $B P$ indicate that the residual has been filtered by a low pass or band pass filter, respectively. As it can be seen in Table I, each of the 3 temporal residuals of wind turbine $i, j$ and extra residual coming from temporal power residual filtered $B P$ is sensitive only to one kind of fault. Then, if a fault is of a size large enough to overpass the uncertainty of the interval temporal models, the effect of this fault in these residuals is distinguishable from the possible effects of other faults even if multiple faults are present in the system.

Moreover, the extra information provided by the spatial residuals increases the robustness in the detectability and isolability, as illustrated in Table II, where the power residuals (LP filtered) of wind column $j$ (wind scenario 1) extracted from (22) are related to the Fault 1 of the wind turbines of this column. If the fault size is not large enough to activate temporal residuals, but activates some spatial residuals, the proposed diagnosis scheme will not always be able to determine the exact wind turbine affected by a fault, but will reduce the candidate faults to some wind turbines in the same wind column. The same problem will occur in the presence of multiple faults in the system.

In total, the theoretical fault signature matrix will be composed by 27 temporal residuals (3 residuals of Table I multiplied by the 9 wind turbines) plus 9 extra residuals coming from the power temporal residuals filtered $B P$. Moreover, if wind case 1 is detected (wind direction close to $0 \mathrm{deg}$ ), 27 spatial residuals (plus 9 extra residuals coming from the power spatial residuals filtered $B P$ ) corresponding to Table II considering the 3 wind columns $(j=1,2,3)$ for pitch angle, angular velocity and power $(L P$ and $B P)$ are considered. If wind case 2 is detected (wind direction close to $45 \mathrm{deg}$ ), 15 spatial residuals (plus 5 extra residuals coming from the power spatial residuals filtered $B P$ ) corresponding to the 3 wind columns defined in (23) are considered.

In order to deal with the effect of non-activated residuals in the matching of the observed fault signatures with the theoretical ones in the isolation procedure, the row-reasoning scheme proposed in [18] has been used. This strategy only considers the residuals that are inconsistent when searching for the fault, since consistency is not relevant. A residual that is found inconsistent indicates that one of the faults that affects the residual is acting on the system. But the contrary is not true, if a residual is satisfied, it does not assure that none of the associated faults is presented.

As uncertainty in spatial residuals is smaller than in temporal residuals, they are activated first when a fault is present in the system. Even in some cases, if the magnitude of the fault is small, spatial residuals are the only ones that are activated. This is the reason why the fault diagnosis algorithm evaluates separately the spatial and temporal residuals of the wind turbines that belong to the same wind column, as illustrated in the fault diagnosis algorithm depicted in Fig. 2.

First, if any temporal or spatial residual related to Fault 1 in wind turbines of wind column 1 (i.e power temporal/spatial residuals filtered $L P$ of the wind turbines that belong to this wind column) is found inconsistent, a fault is detected in the wind turbines of wind column 1. If a fault has been detected, temporal residuals are evaluated, and if one or more temporal residuals are found inconsistent, since every temporal residual is related only to one fault (Table II), the faults related to the residuals found inconsistent are chosen as candidate faults. 
TABLE I

SIGNATURE MATRIX TEMPORAL RESIDUALS WIND TURBINE $i, j$

\begin{tabular}{|c|c|c|c|}
\hline Residuals & $\begin{array}{c}\text { Fault } 1 \\
\text { turbine } i, j\end{array}$ & $\begin{array}{c}\text { Fault 2 } \\
\text { turbine } i, j\end{array}$ & $\begin{array}{c}\text { Fault 3 } \\
\text { turbine } i, j\end{array}$ \\
\hline$r_{T} P_{L P i, j}$ & $\mathrm{x}$ & & \\
\hline$r_{T} P_{B P i, j}$ & & & $\mathrm{x}$ \\
\hline$r_{T} \beta_{L P i, j}$ & & $\mathrm{x}$ & \\
\hline$r_{T} \omega_{g B P i, j}$ & & & $\mathrm{x}$ \\
\hline
\end{tabular}

TABLE II

SigNATURE MATRIX SPATIAL POWER RESIDUALS (LP FILTERED) WIND COLUMN $j\left(0^{\circ}\right)$

\begin{tabular}{|c|c|c|c|}
\hline Residuals & $\begin{array}{c}\text { Fault } 1 \\
\text { turbine } 1, j\end{array}$ & $\begin{array}{c}\text { Fault } 1 \\
\text { turbine } 2, j\end{array}$ & $\begin{array}{c}\text { Fault } 1 \\
\text { turbine } 3, j\end{array}$ \\
\hline$r_{S} P_{L P 1-2, j}$ & $\mathrm{x}$ & $\mathrm{x}$ & \\
\hline$r_{S} P_{L P 1-3, j}$ & $\mathrm{x}$ & & $\mathrm{x}$ \\
\hline$r_{S} P_{L P 2-3, j}$ & & $\mathrm{x}$ & $\mathrm{x}$ \\
\hline
\end{tabular}

Then, the consistency of the candidate faults with the activated spatial residuals is checked and if there is consistency, the faults are correctly isolated. If there is no temporal residual activated, the diagnosis is provided directly by the candidate faults consistent with the activated spatial residuals. If there is no candidate fault consistent with all the activated spatial residuals, the diagnosis will indicate a Fault 1 in wind column 1 but will not specify which wind turbine is affected by Fault 1. This procedure is repeated for the number of wind columns $\left(N_{W D}\right): N_{W D}=3$ in wind scenario $1(0 \mathrm{deg}), N_{W D}=5$ in wind scenario $2(45 \mathrm{deg})$ and $N_{W D}=9$ in other wind scenario (there are not spatial relations available, thus it is considered as many wind columns as many wind turbines in the wind farm). Once Fault 1 has been evaluated for all the wind columns, the same procedure is repeated for Faults 2 and 3.

\section{Results}

In this Section, the results obtained applying the methodR1-1 ologies described above to the wind farm benchmark [33] are presented. The results obtained in wind scenarios 1 and $2(0$ and $45 \mathrm{deg}$ ) are summarized in Table III.

\section{A. Model Calibration}

As a first step, the three different wind speed estimation sequences $\left(\hat{v}_{w_{1}}, \hat{v}_{w_{2}}\right.$ and $\left.\hat{v}_{w_{3}}\right)$ for wind scenario 1 have been computed applying (10) to the wind speed provided by the measuring mast and considering transport delays (10). Fig. 3 shows the real sequence of wind speed in turbine $1,1\left(v_{w_{1,1}}\right)$ and the one estimated for all the turbines in the y-column 1 , i.e. $\hat{v}_{w_{1}}=\hat{v}_{w_{1,1}}=\hat{v}_{w_{2,1}}=\hat{v}_{w_{3,1}}$ (see Fig. 1).

Delay uncertainties $\lambda_{\tau_{1}}, \lambda_{\tau_{2}}$ and $\lambda_{\tau_{3}}$ have been computed as described in Section II-D. $\sigma_{w_{j}}$ and $\sigma_{w_{i, j}}$ bounds have been computed by analysing the error between the wind speed variables and estimations. Functions (13)-(15) have been obtained by means of the physical knowledge of the system and discretized with $T_{s}=0.01 \mathrm{~s}$. Then, the nominal parameters $\theta_{P_{i, j}}^{0}\left(p_{k}\right), \theta_{\beta_{i, j}}^{0}\left(p_{k}\right)$ and $\theta_{\omega_{g i, j}}^{0}\left(p_{k}\right)$ have been obtained and 54 parity equations (27 temporal and 27 spatial) have been built. Applying the $4400 \mathrm{~s}$ long wind sequence described in Section III-A to the Matlab-Simulink $\left.{ }^{(}\right)$benchmark, a

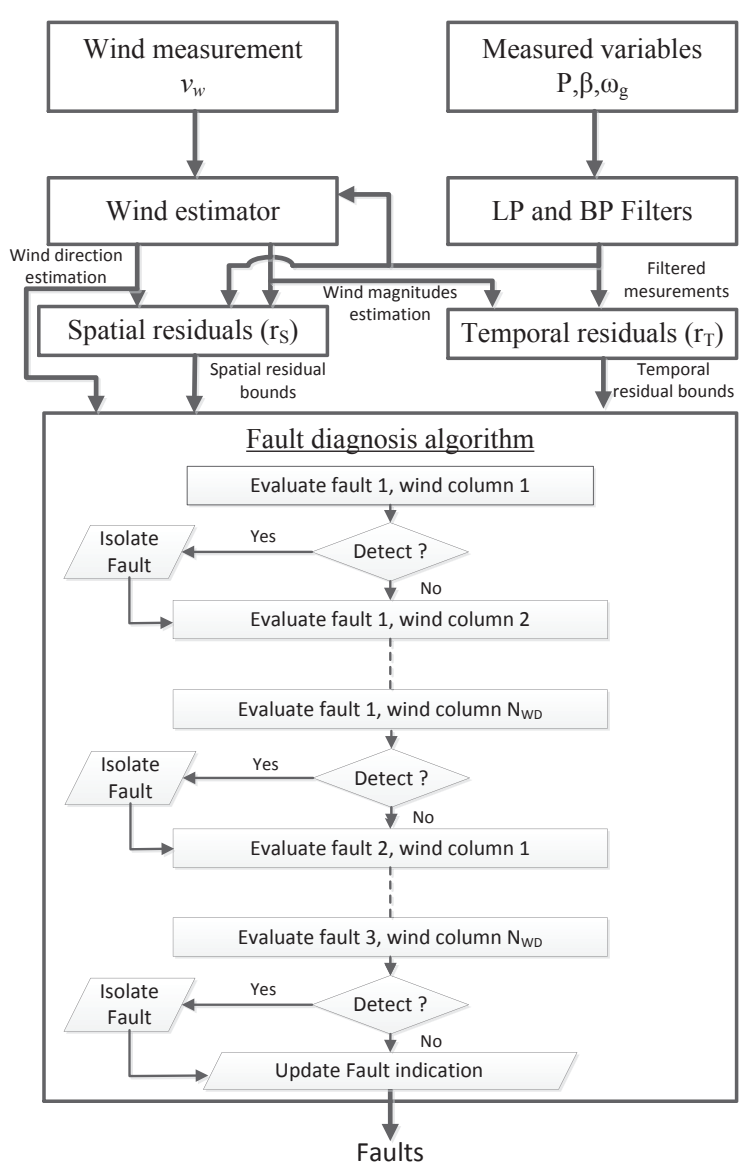

Fig. 2. Scheme of the proposed solution.
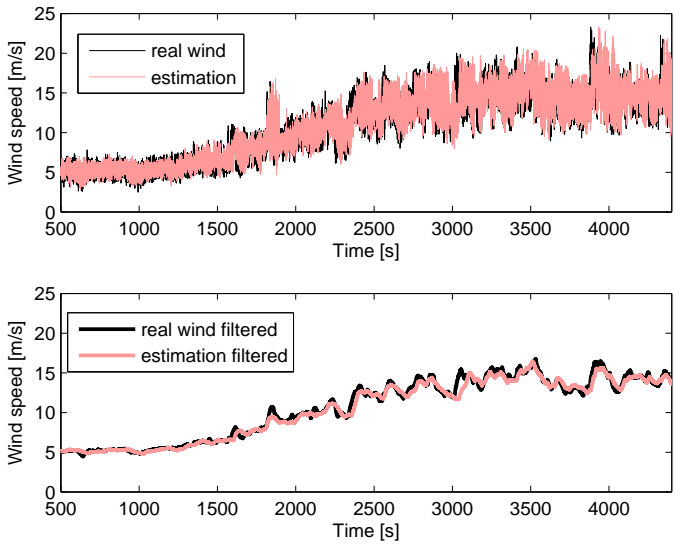

Fig. 3. Real and estimated wind speed in turbine 1,1 .

set of input/output data $P_{r_{i, j}}(k), P_{i, j}(k), \beta_{i, j}(k)$ and $\omega_{g i, j}(k)$, $k=1, \ldots, N$ has been collected. $L P$ and $B P$ filters have been designed in order to minimize the effect of the noise, that has been bounded in each parity equation. Finally, the optimization problem described in Section II-D has been applied, in order to compute the parameter uncertainties $\lambda_{j}(k), j=1, \ldots, n_{\theta}$ for 
each parity equation.

Figs. 4-6 show the evaluation of a residual for each type of magnitude with its interval bounds in the fault free scenario used for model calibration.

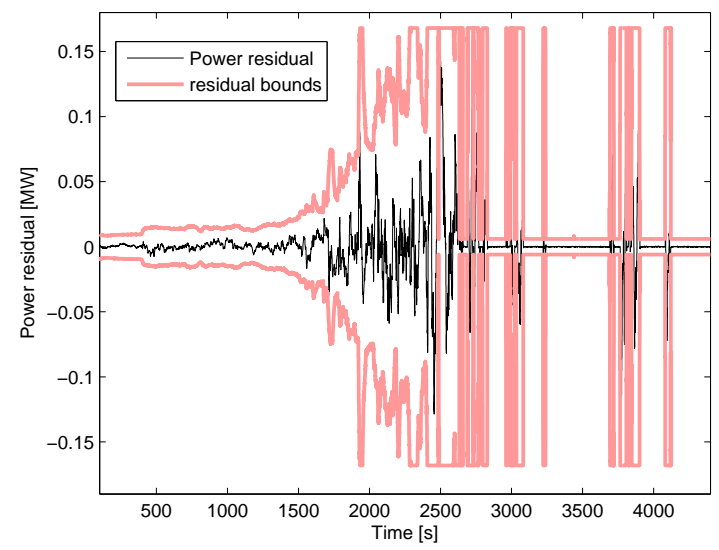

Fig. 4. Residual $r_{S} P_{L P 1-2,2}$ and bounds in fault free scenario.

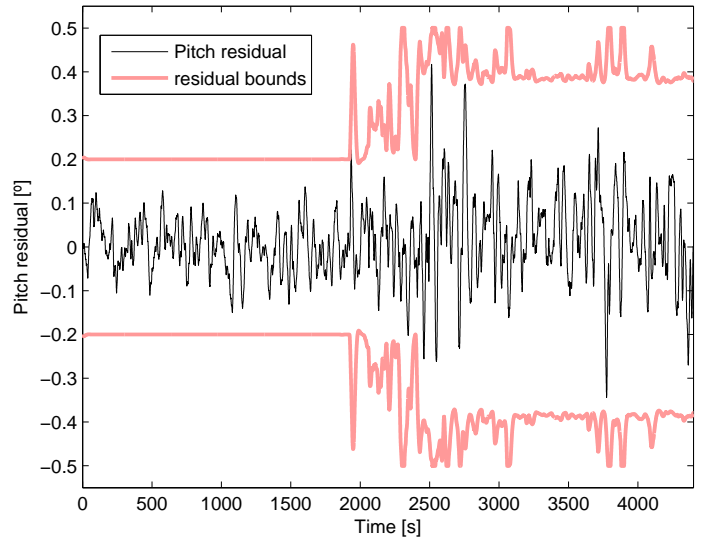

Fig. 5. Residual $r_{S} \beta_{L P 2-3,2}$ and bounds in fault free scenario.

As it can be observed from Fig. 4, the residual interval bounds vary strongly with the operating point in the power spatial residual. This is due to the fact that small differences in the wind speed produce important effects in the power generation. These differences are much lower in the pitch residual (see Fig. 5) and negligible in the angular speed residual (see Fig. 6).

The same procedure has been carried out to calibrate the 42 parity equations (27 temporal and 15 spatial) for wind scenario 2 (45 deg). In this scenario, five different wind speed estimation sequences, that correspond to the number of wind turbine sets that are supposed to receive the same wind speed, have been estimated.

\section{B. Fault Scenarios}

The proposed FDI approach is tested in Fault 1 (the debris build-up on the blades), Fault 2 (pitch misalignment) and Fault 3 (decrease in drive train damping) cases that are described

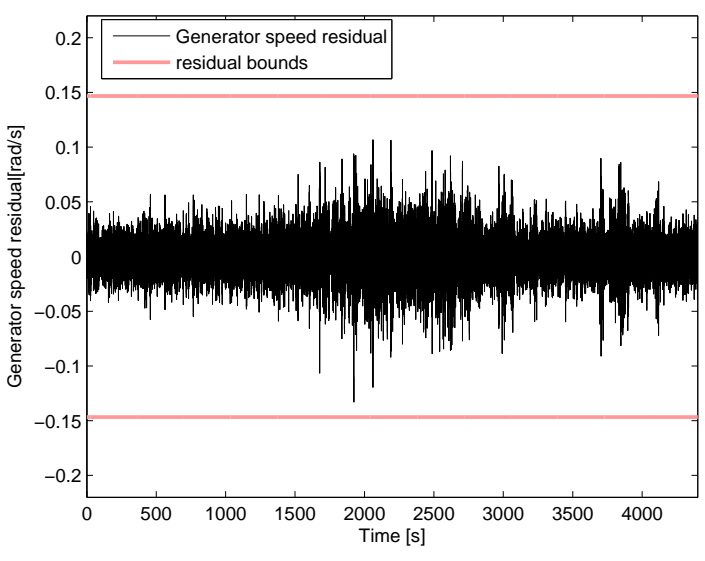

Fig. 6. Residual $r_{S} \omega_{g B P 2-3,2}$ and bounds in fault free scenario.
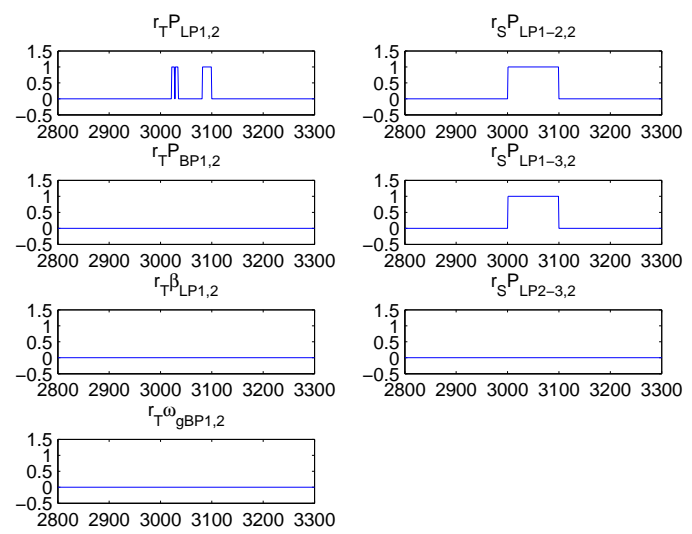

Fig. 7. Inconsistency of residuals related to fault scenario 1(b) (wind scenario $0^{\circ}$ ): Debris build-up in the wind turbine blades lowering maximum power of $8 \%$ in wind turbine 1,2 from $t=3000 \mathrm{~s}$ to $t=3100 \mathrm{~s}$.

in Section III-B. These faults appear twice in three different wind turbines at different time intervals, i.e. no multiple faults are present at any time. All faults occur once before 2300 $\mathrm{s}$ (denoted in the following case $(a)$ ) and once after $2300 \mathrm{~s}$ (denoted in the following case $(b)$ ). In the first period, the wind farm cannot deliver the required power, while it can in the second one.

Figs. 7, 9 and 11 show the evolution of the inconsistency of the residuals related to fault scenarios $1(b)(0 \mathrm{deg}), 3(\mathrm{~b})(0$ $\mathrm{deg})$ and 2(a)(45 deg) respectively. These residuals are the temporal residuals defined in Table I for the turbine $i, j$ affected by each of the above faulty scenarios (turbine 1,2; turbine 3,2 and turbine 1,1 respectively), and the spatial residuals related to the turbine and wind scenario. These are spatial residuals corresponding to the column $j=2$ in Table I for fault scenario $1(\mathrm{~b})(0 \mathrm{deg})$ and fault scenario $3(\mathrm{~b})(0 \mathrm{deg})$ for $\omega_{g}$ spatial residuals filtered $B P$. In the wind scenario of 45 $\mathrm{deg}$, there is no possible spatial redundancy for turbine 1,1 . As it can be noticed from Fig. 7, an inconsistency in spatial residuals $r_{S} P_{L P 1-2,2}$ and $r_{S} P_{L P 1-3,2}$ is detected during all the fault scenario 1(b) and an intermittent inconsistency is detected in residual $r_{T} P_{L P 1,2}$. Other residuals in Fig. 7 and those ones 
that do not appear in Fig. 7 remain consistent during all the fault scenario. Then, applying the row reasoning scheme proposed in [18], the fault in debris build-up on the wind turbine 1,2 is detected and isolated correctly. In a similar way, fault scenarios $3(\mathrm{~b})(0 \mathrm{deg})$ and $2(\mathrm{a})(45 \mathrm{deg})$ are detected and isolated correctly. On the other hand, the evolution of a sensitive residual for each of the three illustrated fault scenarios is depicted in Figs. 8, 10 and 12.

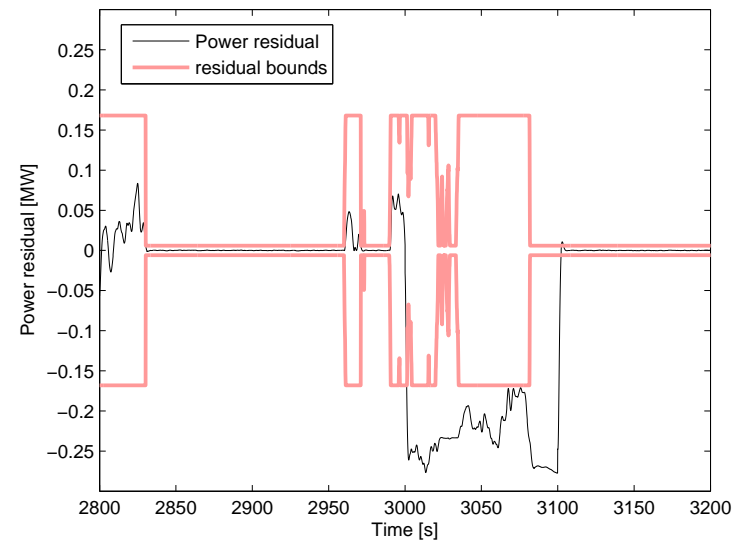

Fig. 8. Evolution of residual $r_{S} P_{L P 1-3,2}$ and bounds in fault scenario 1(b)(wind scenario $0^{\circ}$ ): Debris build-up in the wind turbine blades lowering maximum power of $8 \%$ in wind turbine 1,2 from $t=3000 \mathrm{~s}$ to $t=3100 \mathrm{~s}$.
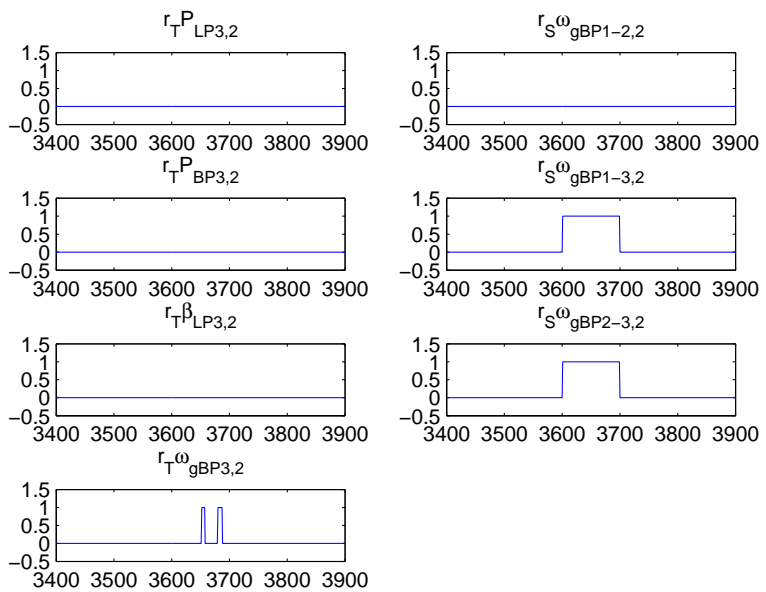

Fig. 9. Inconsistency of residuals related to fault scenario 3(b) (wind scenario $0^{\circ}$ ): decrease in the drive train damping, increase in the amplitude of the sine function by a factor of 4.6 in the wind turbine 3,2 from $t=3600 \mathrm{~s}$ to $t=3700 \mathrm{~s}$.

Table III summarizes the fault detection and isolation results in wind scenarios 1 and 2 of the proposed FDI method implemented in Matlab-Simulink ${ }^{\circledR}$, that have been obtained after an exhaustive Monte Carlo analysis, where the condition of false positive detections proposed in [33] has been verified. For every fault scenario, the magnitude required in order to guarantee the detection i.e., without caring if it is possible to distinguish among some candidate faults in the same wind column), detection/isolation and detection/isolation in less than 3 seconds is presented. In general, the behavior of the FDI

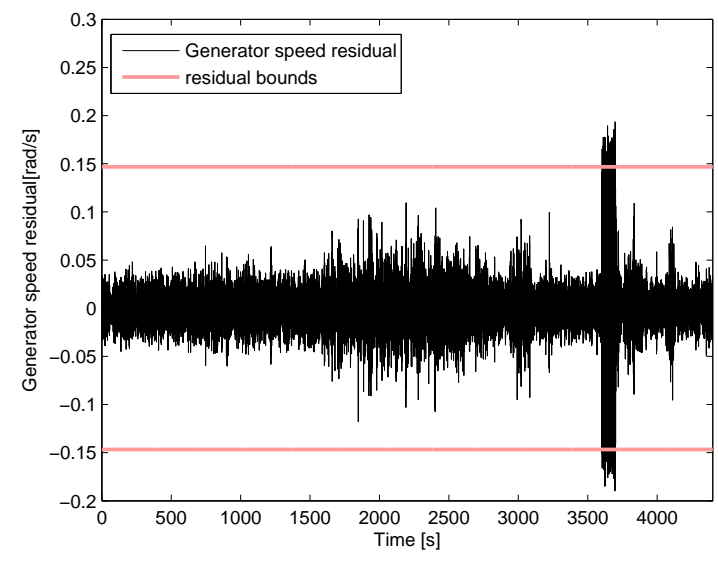

Fig. 10. Evolution of residual $r_{S} \omega_{g B P 2-3,2}$ and bounds in fault scenario 3 (b)(wind scenario $0^{\circ}$ ): decrease in the drive train damping, increase in the amplitude of the sine function by a factor of 4.6 in the wind turbine 3,2 from $t=3600 s$ to $t=3700 s$.
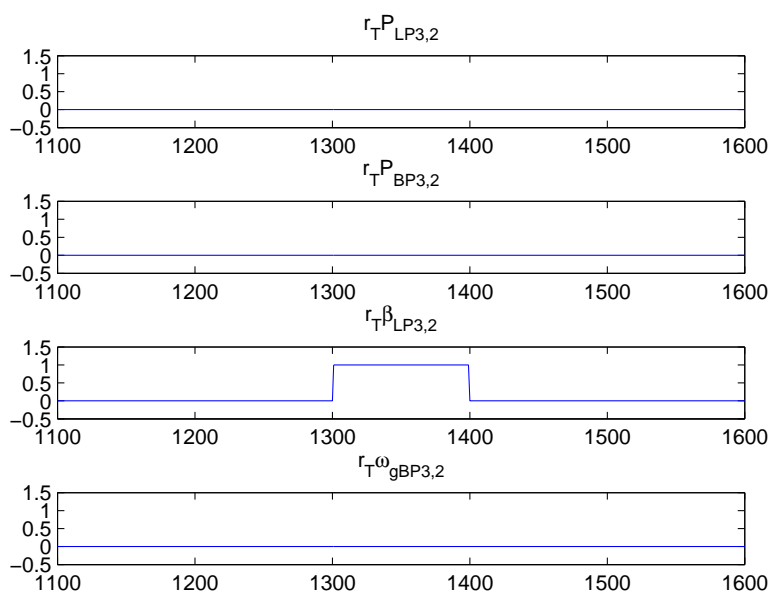

Fig. 11. Inconsistency of residuals related to fault scenario 2(a) (wind scenario $45^{\circ}$ ): misalignment of $0.3^{\circ}$ in the pitch blades of wind turbine 1,1 from $t=1300 \mathrm{~s}$ to $t=1400 \mathrm{~s}$.

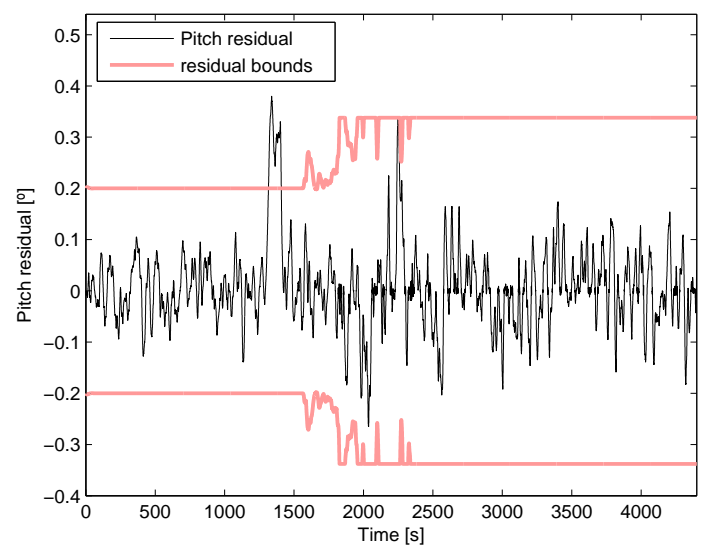

Fig. 12. Evolution of residual $r_{T} \beta_{L P 1,1}$ and bounds in fault scenario 2(a)(wind scenario $45^{\circ}$ ): misalignment of $0.3^{\circ}$ in the pitch blades of wind turbine 1,1 from $t=1300 \mathrm{~s}$ to $t=1400 \mathrm{~s}$. 
method is better in the wind scenario 1 than in wind scenario 2 due to the higher number of spatial relations (27 in wind scenario 1 and 15 in wind scenario 2). Fault 1 (Debris buildup) is detected better in case (b) (after $2300 \mathrm{~s}$ ) due to the fact that the available power is higher and the scaling effect is more important than in case (a) (before $2300 \mathrm{~s}$ ), where the available power is lower. Fault 2 (pitch misalignment) is detected better in case (a) because the uncertainty is slightly lower than in case $(b)$. In order to have a sensitivity of $0.3^{\circ}$ in the fault detection of this fault, a very restrictive low pass filter has been implemented due to the pitch sensor noise. Then, despite the FDI block can detect and isolate $0.3^{\circ}$ in misalignment, the magnitude must be increased to $10^{\circ}-14^{\circ}$ in order to guarantee the correct detection and isolation in less than $3 s$, as proposed by [33]. Finally, Fault 3 (decrease in drive train damping) is detected and isolated correctly [33].

Regarding the computational cost of the solution, the maximum computational cycle time of the whole system (wind farm simulator and proposed FDI solution) implemented in Matlab-Simulink and executed in a laptop computer with a $2.1 \mathrm{GHz}$ Intel Core 2 Duo processor and $2 \mathrm{~GB}$ of RAM memory has always been smaller than the sample time $(0.01 s)$ for all the scenarios studied in the Monte Carlo analysis. The computational cost would be considerably reduced if the FDI algorithm was implemented with enhanced realtime software, e.g. in $\mathrm{C}$ language.

\section{Conclusions}

In this paper, the problem of the fault diagnosis of a wind farm has been addressed using interval parity equations and NLPV models. Fault detection is based on the use of parity equations assuming unknown but bounded description of the noise and modeling errors. The fault detection test is based on checking consistency between the measurements and the model by finding if the measurements are inside the interval prediction bounds. The fault isolation algorithm is based on analyzing the observed fault signatures on-line, and matching them with the theoretical ones obtained using structural analysis. The proposed approach has been tested using the wind farm benchmark proposed in the context of the wind farm FDI/FTC competition obtaining satisfactory results. As future work, the generation of spatial residuals for a generic wind direction, in order to increase the diagnosis robustness, could be considered. Moreover, the design of fault tolerant control strategies, that use the information of the FDI scheme proposed in this paper for accommodating faults in the system, thus minimizing the damage and maximizing the power generated by the wind farm, could be studied.

\section{REFERENCES}

[1] R. Piwko and D. Osborn and R. Gramlich and G. Jordan and D. Hawkins and K. Porter, "Wind energy delivery issues (transmission planning and competitive electricity market operation)," IEEE Power Energy Mag., vol. 3, no. 6, pp. 47-56, 2005.

[2] V. Jalili-Marandi and L.-F. Pak and V. Dinavahi, "Real-time simulation of grid-connected wind farms using physical aggregation," IEEE Trans. Ind. Electron., vol. 57, no. 9, pp. 3010-3021, 2010.

[3] S. Chuangpishit and A. Tabesh and Z. Moradi-Shahrbabak and M. Saeedifard, "Topology design for collector systems of offshore wind farms with pure DC power systems," IEEE Trans. Ind. Electron., vol. 61, no. 1 , pp. $320-328,2014$.
TABLE III

FAult Detection IsOlation Results (AMP. REFERS TO THE AMPLITUDE IN THE DRIVE TRAIN OSCILLATION)

\begin{tabular}{|c|c|c|c|}
\hline Fault & $0^{\circ} / 45^{\circ}$ & Magnitude & Detection/Isolation \\
\hline 1(a) & $0^{\circ}$ & $\begin{array}{l}\geq 7 \% \\
\geq 8 \% \\
\geq 14 \% \\
\end{array}$ & $\begin{array}{c}\text { Detection } \\
\text { Detection and Isolation } \\
\text { Detect. and Isol. } \leq 3 s\end{array}$ \\
\hline 1(a) & $45^{\circ}$ & $\begin{array}{l}\geq 10 \% \\
\geq 12 \% \\
\geq 14 \%\end{array}$ & $\begin{array}{c}\text { Detection } \\
\text { Detection and Isolation } \\
\text { Detect. and Isol. } \leq 3 s\end{array}$ \\
\hline 1(b) & $0^{\circ}$ & $\begin{array}{l}\geq 3 \% \\
\geq 4 \% \\
\geq 7 \%\end{array}$ & $\begin{array}{c}\text { Detection } \\
\text { Detection and Isolation } \\
\text { Detect. and Isol. } \leq 3 \mathrm{~s}\end{array}$ \\
\hline 1(b) & $45^{\circ}$ & $\begin{array}{l}\geq 3 \% \\
\geq 6 \% \\
\geq 12 \%\end{array}$ & $\begin{array}{c}\text { Detection } \\
\text { Detection and Isolation } \\
\text { Detect. and Isol. } \leq 3 \mathrm{~s}\end{array}$ \\
\hline 2(a) & $0^{\circ}$ & $\begin{array}{l}\geq 0.3^{\circ} \\
\geq 0.3^{\circ} \\
\geq 3.6^{\circ} \\
\end{array}$ & $\begin{array}{c}\text { Detection } \\
\text { Detection and Isolation } \\
\text { Detect. and Isol. } \leq 3 s\end{array}$ \\
\hline 2(a) & $45^{\circ}$ & $\begin{array}{l}\geq 0.3^{\circ} \\
\geq 0.3^{\circ} \\
\geq 14^{\circ}\end{array}$ & $\begin{array}{c}\text { Detection } \\
\text { Detection and Isolation } \\
\text { Detect. and Isol. } \leq 3 s\end{array}$ \\
\hline 2(b) & $0^{\circ}$ & $\begin{array}{l}\geq 0.4^{\circ} \\
\geq 0.6^{\circ} \\
\geq 10^{\circ}\end{array}$ & $\begin{array}{c}\text { Detection } \\
\text { Detection and Isolation } \\
\text { Detect. and Isol. } \leq 3 s\end{array}$ \\
\hline 2(b) & $45^{\circ}$ & $\begin{array}{l}\geq 0.3^{\circ} \\
\geq 0.3^{\circ} \\
\geq 8^{\circ}\end{array}$ & $\begin{array}{c}\text { Detection } \\
\text { Detection and Isolation } \\
\text { Detect. and Isol. } \leq 3 \mathrm{~s}\end{array}$ \\
\hline $3(\mathrm{a})$ & $0^{\circ}$ & $\begin{array}{c}\text { Amp. } \geq 2.3 \\
\text { Amp. } \geq 2.3 \\
\text { Amp. } \geq 3\end{array}$ & $\begin{array}{c}\text { Detection } \\
\text { Detection and Isolation } \\
\text { Detect. and Isol. } \leq 3 \mathrm{~s}\end{array}$ \\
\hline $3(\mathrm{a})$ & $45^{\circ}$ & $\begin{array}{l}A m p . \geq 2.3 \\
\text { Amp. } \geq 2.3 \\
\text { Amp. } \geq 3.5\end{array}$ & $\begin{array}{c}\text { Detection } \\
\text { Detection and Isolation } \\
\text { Detect. and Isol. } \leq 3 \mathrm{~s}\end{array}$ \\
\hline 3(b) & $0^{\circ}$ & $\begin{array}{l}\text { Amp. } \geq 2.3 \\
\text { Amp. } \geq 2.3 \\
\text { Amp. } \geq 2.8\end{array}$ & $\begin{array}{c}\text { Detection } \\
\text { Detection and Isolation } \\
\text { Detect. and Isol. } \leq 3 \mathrm{~s}\end{array}$ \\
\hline 3(b) & $45^{\circ}$ & $\begin{array}{l}\text { Amp. } \geq 2.3 \\
\text { Amp. } \geq 2.3 \\
\text { Amp. } \geq 2.4\end{array}$ & $\begin{array}{c}\text { Detection } \\
\text { Detection and Isolation } \\
\text { Detect. and Isol. } \leq 3 \mathrm{~s}\end{array}$ \\
\hline
\end{tabular}

[4] G. M. Joselin Herbert and S. Iniyan and R. Goic, "Performance, reliability and failure analysis of wind farm in a developing country," Renew. Energ., vol. 35, no. 12, pp. 2739-2751, 2010.

[5] I. El-Thalji and E. Jantunen, "On the development of condition based maintenance strategy for offshore wind farm: requirement elicitation process," Energy Procedia, vol. 24, pp. 328-339, 2012.

[6] G. R. Biswal and R. P. Maheshwari and M. L. Dewal, "Dynamic process control and monitoring of novel $S^{3} R S$ based hydrogen cooling system," Int. J. Elec. Power, vol. 43, no. 1, pp. 162-172, 2012.

[7] G. R. Biswal and R. P. Maheshwari and M. L. Dewal, "System reliability and fault tree analysis of SeSHRS-based augmentation of hydrogen: dedicated for combined cycle power plants," IEEE Syst. J., vol. 6, no. 4, pp. 647-656, 2012.

[8] E. Duviella, L. Serir, and M. Sayed-Mouchaweh, "An evolving classification approach for fault diagnosis and prognosis of a wind farm," in 2nd Conf. on Control and Fault-Tolerant Systems (SysTol), Nice, France, 2013, pp. 377-382.

[9] S. Karimi, A. Gaillard, P. Poure, and S. Saadate, "FPGA-based real-time power converter failure diagnosis for wind energy conversion systems," IEEE Trans. Ind. Electron., vol. 55, no. 12, pp. 4299-4308, 2008.

[10] W. Yang, P. Tavner, C. Crabtree, and M. Wilkinson, "Cost-effective condition monitoring for wind turbines," IEEE Trans. Ind. Electron., vol. 57, no. 1, pp. 263-271, 2010 .

[11] X. Gong and W. Qiao, "Bearing fault diagnosis for direct-drive wind turbines via current-demodulated signals," IEEE Trans. Ind. Electron., vol. 60, no. 8, pp. 3419-3428, 2013.

[12] N. M. A. Freire and J. O. Estima and A. J. Marques Cardoso, "Opencircuit fault diagnosis in PMSG drives for wind turbine applications," IEEE Trans. Ind. Electron., vol. 60, no. 9, pp. 3057-3967, 2013.

[13] P. F. Odgaard and J. Stoustrup and M. Kinnaert, "Fault tolerant control of wind turbines - a benchmark model," IEEE Trans. Control Syst. Technol., vol. 21, no. 4, pp. 1168-1182, 2013.

[14] W. Chen, S. X. Ding, A. Abandan Haghani, A. Naik, A. Q. Khan, and 
S. Yin, "Observer-based FDI schemes for wind turbine benchmark," in Proc. 18th IFAC World Congress, Milan, Italy, 2011, pp. 7073-7078.

[15] S. Simani, P. Castaldi, and A. Tilli, "Data-driven approach for wind turbine actuator and sensor fault detection and isolation," in Proc. 18th IFAC World Congress, Milan, Italy, 2011, pp. 8301-8306.

[16] C. Svärd and M. Nyberg, "Automated design of an FDI system for the wind turbine benchmark," Journal of Control Science and Engineering, vol. 2012, pp. 1-13, 2012.

[17] F. Stoican, C.-F. Raduinea, and S. Olaru, "Adaptation of set theoretic methods to the fault detection of a wind turbine benchmark," in Proc. 18th IFAC World Congress, Milan, Italy, 2011, pp. 8322-8327.

[18] J. Blesa and D. Rotondo and V. Puig and F. Nejjari, "FDI and FTC of wind turbines using the interval observer approach and virtual actuators/sensors," Control Eng. Pract., vol. 24, pp. 138-155, 2014.

[19] J. Gertler, Fault detection and diagnosis in engineering systems. New York: Marcel Dekker, 1998

[20] J. Chen and R. J. Patton, Robust model-based fault diagnosis for dynamic systems. Kluwer Academic Publishers, 1999.

[21] M. Blanke, M. Kinnaert, J. Lunze, and M. Staroswiecki, Diagnosis and fault-tolerant control. Springer-Verlag Berlin Heidelberg, 2006.

[22] R. Isermann, "Model-based fault-detection and diagnosis - status and applications," Аппи. Rev. Control, vol. 29, no. 1, pp. 71-85, 2005.

[23] F. Previdi and M. Lovera, "Identification of non-linear parametrically varying models using separable least squares," Int. J. Control, vol. 77, no. 16, pp. 1382-1392, 2004.

[24] T. Takagi and M. Sugeno, "Fuzzy identification of systems and its applications to modeling and control," IEEE Trans. Syst., Man, Cybern., vol. SMC-15, no. 1, pp. 116-132, 1985.

[25] R. Babuška, Fuzzy modeling for control. Kluwer, Boston, MA, 1998.

[26] P. M. Mäkilä and P. Viljamaa, "Convex Parametric Design, Gain Scheduling, and Fuzzy Computing," Institute of Automation and Control, Tampere University of Technology, Tampere, Finland, Tech. Rep., 2002.

[27] P. Bergsten, R. Palm, and D. Driankov, "Observers for Takagi-Sugeno fuzzy systems," IEEE Trans. Syst., Man, Cybern., B, Cybern., vol. 32, no. 1, pp. 114-121, 2002.

[28] Q. Rong and G. W. Irwin, "LMI-Based Control Design for Discrete Polytopic LPV Systems," in Proc. 6th European Control Conference (ECC), Cambridge, UK, 2003.

[29] V. Puig, A. Stancu, T. Escobet, F. Nejjari, J. Quevedo, and R. J. Patton, "Passive robust fault detection using interval observers: application to the DAMADICS benchmark problem," Control Eng. Pract., vol. 14, 2006.

[30] V. Puig, J. Quevedo, T. Escobet, F. Nejjari, and S. de las Heras, "Passive robust fault detection of dynamic processes using interval models," IEEE Trans. Control Syst. Technol., vol. 16, pp. 1083-1089, 2008.

[31] I. Fagarasan, S. Ploix, and S. Gentil, "Causal fault detection and isolation based on a set-membership approach," Automatica, pp. 2099-2110, 2004.

[32] M. A. Sainz, J. Armengol, and J. Vehí, "Fault detection and isolation of the three-tank system using the modal interval analysis," J. Process Contr., vol. 12, no. 2, pp. 325-338, 2002.

[33] P. F. Odgaard and J. Stoustrup, "Fault tolerant wind farm control - a benchmark model," in Proc. 8th IEEE Multi-conference on Systems and Control (MSC), Hydebarad, India, 2013, pp. 412-417.

[34] R. J. Patton and G. P. Liu, "Robust control design via eigenstructure assignment, genetic algorithms and gradient-based optimization," IEE P.-Contr. Theor. Ap., vol. 141, no. 3, pp. 202-208, 1994.

[35] L. Jaulin, M. Kieffer, O. Didrit, and E. Walter, Applied interval analysis. Springer, 2001.

[36] J. Blesa and V. Puig and Y. Bolea, "Fault detection using interval LPV models in an open-flow canal," Control Eng. Pract., vol. 18, no. 5, pp. 460-470, 2010.

[37] J. F. Bard, Practical bilevel optimization: algorithms and applications, ser. Nonconvex optimization and its applications, vol. 30. Springer, 1998.

[38] P. F. Odgaard, M. Baekgaard, and B. Astrup, "Model based control of wind parks," in Proc. European Wind Energy Conference (EWEC), Warsaw, Poland, 2010.

[39] K. E. Johnson and L. Y. Pao and M. J. Balas and L. J. Fingersh, "Control of variable-speed wind turbines: standard and adaptive techniques for maximizing energy capture," IEEE Control Syst. Mag, vol. 26, no. 3, pp. 70-81, 2006.

[40] P. F. Odgaard and J. Stoustrup, "Results of a wind turbine FDI competition," in Proc. 8th IFAC Symposium SAFEPROCESS, Mexico City, Mexico, 2012, pp. 102-107.
[41] A. J. Brand and J. W. Wagehaar, "A quasi-steady wind farm flow model in the context of distributed control of the wind farm," in Proc. European Wind Energy Conference (EWEC), Warsaw, Poland, 2010, pp. 202-205. 